\title{
Transformation Magnetohydrodynamics in Presence of a Channel Filled with Porous Medium and Heat Transfer of Non-Newtonian Fluid by Using Lie Group Transformations
}

\author{
Nazek Alessa \\ Mathematical Sciences, Faculty of Science, Princess Nourah Bint Abdulrahman University, Saudi Arabia \\ Correspondence should be addressed to Nazek Alessa; nazekaa@yahoo.com
}

Received 29 August 2020; Revised 23 September 2020; Accepted 26 September 2020; Published 22 October 2020

Academic Editor: Maria Alessandra Ragusa

Copyright ( $\odot 2020$ Nazek Alessa. This is an open access article distributed under the Creative Commons Attribution License, which permits unrestricted use, distribution, and reproduction in any medium, provided the original work is properly cited.

In this paper, the numerical results are presented by using Lie group transformations, to be more efficient and sophisticated. To solve various fluid dynamic problems numerically, we present the numerical results in a field of velocity and distribution of temperature for different parameters regarding the problem of radiative heat, a magnetohydrodynamics, and non-Newtonian viscoelasticity for the unstable flow of optically thin fluid inside a channel filled with nonuniform wall temperature and saturated porous medium, including Hartmann number, porous medium and frequency parameter, and radiation parameter, with a comparison of the corresponding flow problems for a Newtonian fluid. Moreover, the effects of the pertinent parameters on the friction coefficient of skin and local Nusselt number were discussed numerically and also illustrate that graphically.

\section{Introduction}

The electromagnetic propulsion is an important application of magnetohydrodynamic flow of non-Newtonian viscoelastic fluids. Also, the magnetohydrodynamics is one of the applications of an electrically conducting fluid, as well as it will help the fluids which have thixotropic behavior in the flow of the liquid as the blood, lubrication with greases and heavy oils, and plastic extension. They have applications in chemical engineering as well.

Lie group transformation was developed $[1,2]$ to use in mechanics of fluid and also in transformations of equations to solve some nonlinear problems.

Several years ago, simple flow problems in hydrodynamics were study, and new attention has been given to the magnetohydrodynamics by Moreau [3]. Control transfer unsteadies the heat in the vertical porous channel [4]. There are many applications for the motion of Newtonian fluids in many areas, such as the flow and heat transfer magnetohydrodynamics, the nuclear fuel flow, flow of blood, and also by using the multideck asymptotic technique in Poiseuille flow [5-9]. Considering the electromagnetic propulsion is another important application. This system is consists of plasma and source of power (such as a reactor of nuclear) and also tube in which the electromagnetic forces accelerate the plasma. In studying such a system, it requires understanding the properties of transport and state equation, the relationship of shear rate-shear stress, the conductivity of electrical, thermal conductivity, and radiation. It is worth to mention that this study is closely associated with magnetochemistry.

The presence of an external magnetic field will likely affect some of those properties where the plasma sets in motion of hydrodynamic $[10,11]$.

In the last years, researchers have been interested in the issue of the flow of fluid through porous media, because the pores of the reservoir rocks recover the crude oil and at the same time because of the magnetic field effect on the work and quality of the systems. There are several studies on the transfer of heat in porous media and the flow of magnetohydrodynamics [12]. Chamkha [13] has considered the effects of Hall on a vertical plate during the flow of magnetohydrodynamic free convection through a thermally stratified porous medium. For instance, Raptis et al., in the presence of a porous medium through two parallel panels, [14] have analyzed the flow of hydromagnetic free convection [15] 
through vertical plate and studied the flow of mixed convection in a porous medium under the effect of a magnetic field. Through a porous medium in a channel filled with it, Makinde et al. [16] have studied the possibility of heat transfer to the magnetohydrodynamic flow inside the cannel.

The present paper is aimed at modifying the study of Makinde et al. [16] for non-Newtonian fluid and investigating the combined effects of non-Newtonian viscoelasticity and radiative heat transfer through a channel filled with porous medium for optically thin fluid. In this work, we have used Tanhed method to transform a system of partial differential equations to a system of nonlinear ordinary differential equations, and then solve it numerically by using the shooting method. Numerical results were present for skin friction coefficient, velocity profile, temperature distribution, and local Nusselt number, with a comparison of the corresponding flow problems for a Newtonian fluid. The effects of the different parameters of the problem on the flow have been shown and discussed graphically.

\section{Mathematical Analysis}

Assume that the unsteady flow of non-Newtonian fluid of conducting an optically thin fluid in a porous medium inside a channel filled with it, under the influence of radiative heat transfer and field of homogeneous magnetic an externally applied (see Figure 1).

Suppose that the force of electromagnetic produced is too small and the fluid has a weak electrical conductivity. Let $(a, b)$ system of Cartesian, such that $a$ lies along the center of the channel, and $b$ is the distance measured. Let a model of Boussinesq incompressible fluid, then give as the equations of governing the motion.

$$
\begin{aligned}
\frac{\partial z}{\partial i} & =-\frac{1}{\lambda} \frac{\partial u}{\partial a}+\vartheta \frac{\partial^{2} z}{\partial b^{2}}+v \frac{\partial^{3} z}{\partial i \partial b^{2}}-\left(\frac{\vartheta}{P}-\frac{\rho E_{0}^{2}}{\lambda}\right) z+h \alpha r-h \alpha r_{0}, \\
\frac{\partial r}{\partial i} & =\frac{t}{\lambda S} \frac{\partial^{2} r}{\partial b^{2}}-\frac{1}{\lambda S} \frac{\partial v}{\partial b} .
\end{aligned}
$$

The problem's boundary conditions are

$$
\begin{array}{lll}
z=0, & r=r_{j} & \text { at } b=m, \\
z=0, & r=r_{0} & \text { at } b=0 .
\end{array}
$$

Let the temperatures $r_{0}, r_{j}$ of both walls are high enough to transfer heat of radiative. [17] Cogley et al. supposed that the optically thin fluid has a relatively low density with a flux of radiative heat shown as follows:

$$
\frac{\partial v}{\partial i}=4 \phi^{2}\left(r_{0}-r_{j}\right)
$$

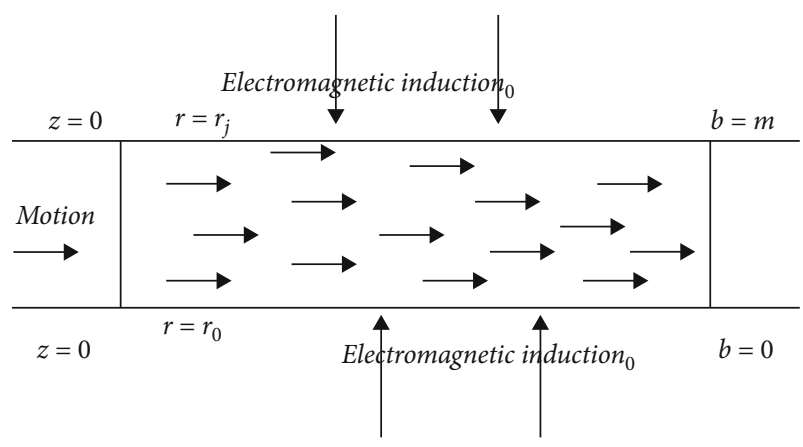

Figure 1: Described the problem.

such that $\phi$ is the mean coefficient of radiation absorption. Now, introducing these dimensionless variables,

$$
\bar{a}=\frac{a}{m}, \bar{b}=\frac{b}{m}, \bar{z}=\frac{z}{w}, \eta=\frac{r-r_{0}}{r_{j}-r_{0}}, i^{\prime}=\frac{i w}{m}, \bar{u}=\frac{m u}{\lambda \vartheta w},
$$

such that $w$ is the flow, and in another meaning, it is velocity. The governing equations of dimensionless with ignoring the bars (for clarity) been given by

$$
\begin{aligned}
& y \frac{\partial \bar{z}}{\partial i^{\prime}}=-\frac{\partial \bar{u}}{\partial \bar{a}}+\frac{\partial^{2} \bar{z}}{\partial \bar{b}^{2}}+A \frac{\partial^{3} \bar{z}}{\partial i^{\prime} \partial \bar{b}^{2}}-q \bar{z}-N \bar{z}+f_{g} \eta, \\
& L \frac{\partial \eta}{\partial i^{\prime}}=\frac{\partial^{2} \eta}{\partial \bar{b}^{2}}+R \eta .
\end{aligned}
$$

The transformed boundary conditions may be written as follows:

$$
\begin{array}{lll}
\bar{z}=0, & \eta=1 \quad \text { at } b=1, \\
\bar{z}=0, & \eta=0 & \text { at } b=0,
\end{array}
$$

where the dimensionless parameters are defined as follows:

$$
\begin{aligned}
\text { Grashof number }: f_{g} & =\frac{h \alpha r_{j} m^{2}-h \alpha r_{0} m^{2}}{\nu U}, \\
\text { Hartmann number }: N & =\frac{m^{2} \rho E^{2}}{\rho \vartheta}, \\
\text { Radiation parameter }: R & =\frac{4 \phi^{2} m^{2}}{t}, \\
\text { Peclet number }: L & =\frac{w m \lambda S}{t}, \\
\text { Darcy number }: C & =\frac{P}{m^{2}},
\end{aligned}
$$

Porous medium shape factor parameter $: q=\frac{1}{C}$,

$$
\text { Non-Newtonian (viscoelasticity) parameter : } A=\frac{v w}{m \vartheta} \text {. }
$$




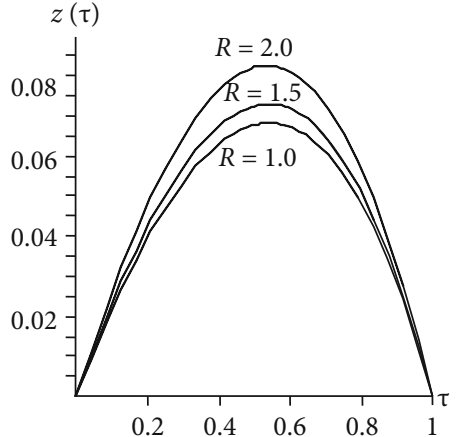

Figure 2: Velocity profile for various values of $R$ with $q=1.0$, $N=1.0, A=1.0$, and $j i^{\prime}=\pi / 4$.

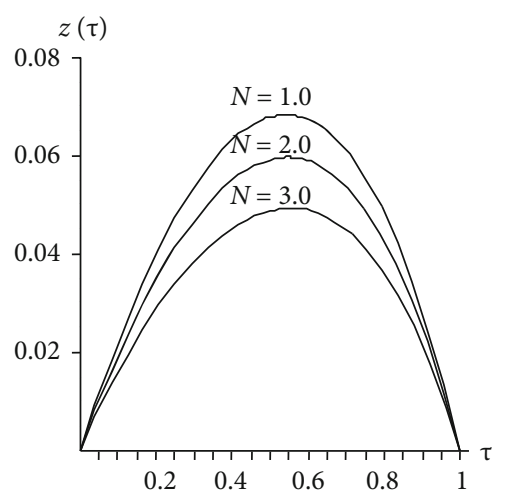

FIGURE 3: Velocity profile for various values of $N$ with $q=1.0$, $R=1.0, A=1.0$, and $j i^{\prime}=\pi / 4$.

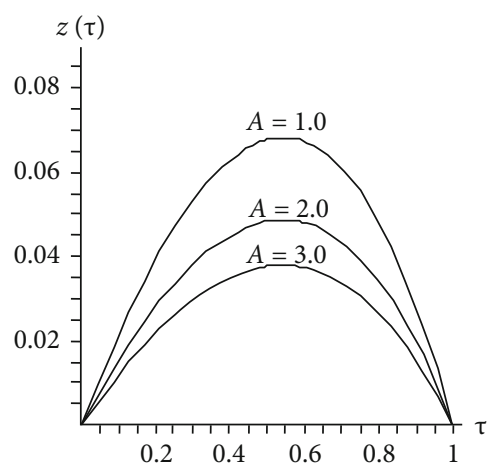

Figure 4: Velocity profile for various values of $A$ with $q=1.0$, $N=1.0, R=1.0$, and $j i^{\prime}=\pi / 4$.

2.1. Lie Group Transformations. We are using this method to solve the problems of fluid dynamics numerically, with efficiently and high quality [18].

Let

$-\frac{\partial \bar{u}}{\partial \bar{a}}=\mu \tanh \left(j i^{\prime}\right), \quad \bar{z}\left(\bar{b}, i^{\prime}\right)=z_{0}(\bar{b}) \tanh \left(j i^{\prime}\right), \quad \eta\left(\bar{b}, i^{\prime}\right)=\eta_{0}(\bar{b}) \tanh \left(j i^{\prime}\right)$,

such that $\mu$ is a constant and $j$ is the oscillation frequency.

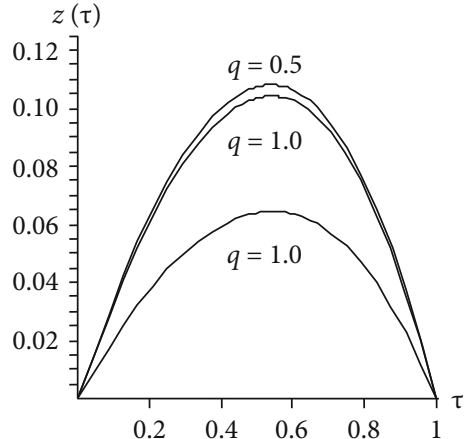

FIgURE 5: Velocity profile for various values of $q$ with $N=1.0$, $R=1.0, A=1.0$, and $j i^{\prime}=\pi / 4$.

2.2. Solution Mechanism. To solve equations (5) and (6) under the boundary condition (7), replace the expressions above in equation (9) into equations (5) to (7), and we obtain

$$
\begin{aligned}
& \frac{d^{2} z_{0}}{d \bar{b}^{2}}-n_{1} z_{0}=-n_{2}\left(\mu+f_{g} \eta_{0}\right), \\
& \frac{d^{2} \eta_{0}}{d \bar{b}^{2}}+n_{3} \eta_{0}=0,
\end{aligned}
$$

with boundary conditions

$$
\begin{aligned}
& z_{0}=0, \quad \eta_{0}=\operatorname{coth}\left(j i^{\prime}\right) \quad \text { at } \bar{b}=1, \\
& z_{0}=0, \quad \eta_{0}=0 \quad \text { at } \bar{b}=0,
\end{aligned}
$$

where $n_{1}=q+N+2 j y \operatorname{cosech}\left(2 j i^{\prime}\right) / 1+2 A j \cos \operatorname{ech}\left(2 j i^{\prime}\right)$, $n_{2}=1 / 1+2 A j \operatorname{cosech}\left(2 j i^{\prime}\right)$, and $n_{3}=R-2 j L \operatorname{cosech}\left(2 j i^{\prime}\right)$.

2.3. Skin Friction and Nusselt Number. The upper wall of the channel defines the shear stress (13) more clearly; is $\delta$ considered in the case that $b=0$ ?

$$
\delta=\left(\frac{w}{m} \frac{\partial \bar{z}}{\partial \bar{b}}+\frac{\gamma_{1} w^{2}}{\gamma m^{2}} \frac{\partial^{2} \bar{z}}{\partial i^{\prime} \partial \bar{b}}\right)_{b=0} .
$$

The local nondimensional skin friction coefficient $\psi$ is given by

$$
\psi=\frac{\delta}{w / m}=\tanh \left(j i^{\prime}\right) z_{0}^{\prime}(0)+\varepsilon \sec \zeta^{2}\left(j i^{\prime}\right) z_{0}^{\prime \prime}(0)
$$

where $\varepsilon=\gamma_{1} w j / \gamma m$.

The heat transfer rate is defined as follows:

$$
v_{j}=-\left.t \frac{\partial r}{\partial \bar{b}}\right|_{b=0} .
$$

The coefficient of local heat transfer is defined as follows:

$$
\zeta(\bar{b})=\frac{m v_{j}(\bar{b})}{r_{j}-r_{0}}
$$




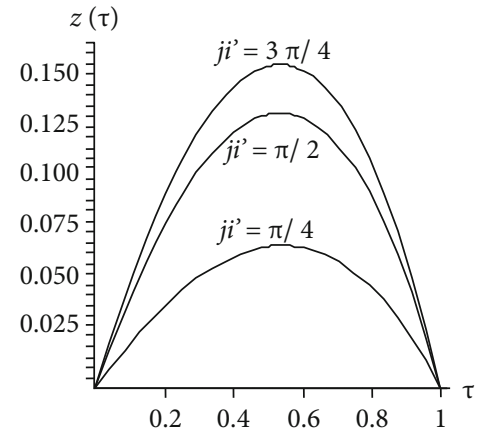

FIGURE 6: Velocity profile for various values of $j i^{\prime}$ with $q=1.0$, $N=1.0, R=1.0$, and $A=1.0$.

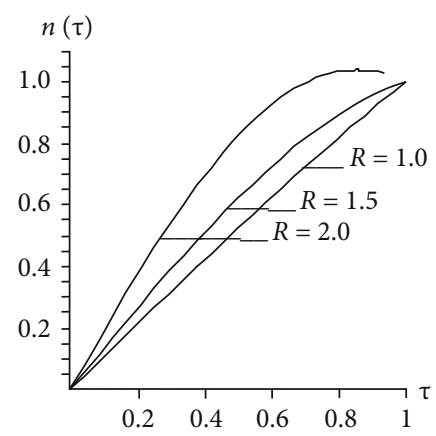

FIGURE 7: Fluid temperature for various values of $R$ with $q=1.0$, $N=1.0, A=1.0$, and $j i^{\prime}=\pi / 4$.

The local Nusselt number may be written as

$$
R_{z}=\frac{\zeta(\bar{b})}{t}=-\tanh \left(j i^{\prime}\right) \eta_{0}^{\prime}(0)
$$

\section{Results and Discussion}

We used fourth-order Runge-Kutta integration and Newton's shooting method to solve equations (10) and (11) under the boundary conditions (12) numerically, for different values of the parameters, and used the following parameter values $L=0.71, f_{g}=1.0, \quad y=1.0, \quad \mu=1.0, \quad$ and $j=1.0$. Figures $2-6$, we noticed the profile of velocity for various values in the parameter of the radiation $R$, Hartmann number $N$, non-Newtonian (viscoelasticity) parameter $A$, porous medium parameter $q$, and frequency parameter $j i^{\prime}$. In Figures 2 and 3, we can notice that the profile of fluid velocity is parabolic where the minimum value concentrated at the walls, and the maximum value concentrated along the centerline of the channel. Nevertheless, we can observe that the fluid velocity profile decreases when the Hartmann number is increased and increases if the radiation parameter is increased, which means a magnetic field intensity. Figures 4 and 5 clearly show that the fluid velocity decreases when there is an increase in a porous medium and increases when there is a decrease in the non-Newtonian (viscoelasticity) parameter. In Figure 6, we can note that the fluid velocity increases when there is an increase in the frequency parame-

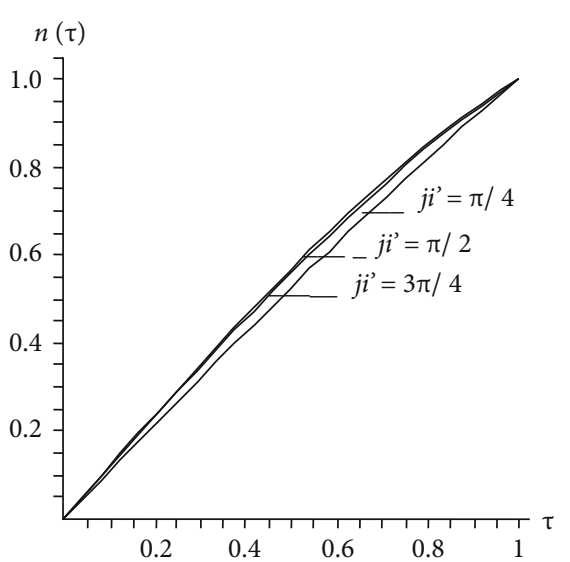

FIgURE 8: Fluid temperature for various values of $j i^{\prime}$ with $q=1.0$, $N=1.0, R=1.0$, and $A=1.0$.

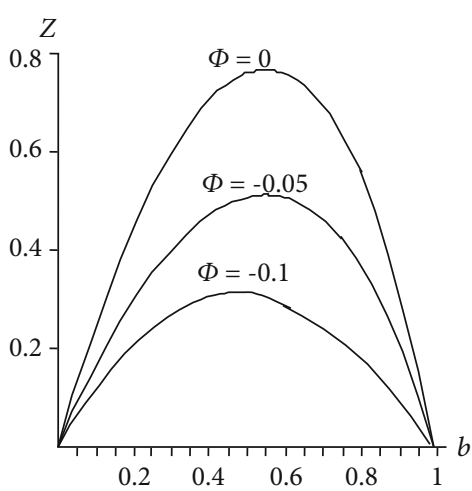

Figure 9: Velocity of $Z$ when $N=0.5, R=1.5$.

ter. In Figures 7 and 8, we can note that the fluid temperature increases when there is an increase in the radiation parameter and increases when there is a decrease in the frequency parameter.

The magnetic field can produce a resistive type force that appears to withstand the fluid flow, and its velocity reduces, where radiation parameter value $R$ increases with fixed magnetic field parameter $N$ values (see Figures 9 and 10).

Table 1 illustrates the effects of $A, N, q, R$, and $j i{ }^{\prime}$ on the coefficient of skin friction (wall shear stress) and the number of local Nusselt (wall heat transfer rate).

Coefficient decreases as $A, q$, and $N$ increase but increases as $R$ and $j i^{\prime}$ increase, and the local Nusselt number increases as $R$ and $j i^{\prime}$ increase.

\section{Conclusion}

This paper reviewed the combined effects of a transverse magnetic field, non-Newtonian viscoelasticity, and radiative heat transfer to unsteady the flow of optically thin fluid in a porous medium inside a channel filled with it and nonuniform channel wall temperature. This paper presented the results of the temperature and velocity numerically and then used to compute the rate's transfer of heat at the channel walls and shear stress of the wall. In general, these results show that the increase in non-Newtonian fluid parameter 


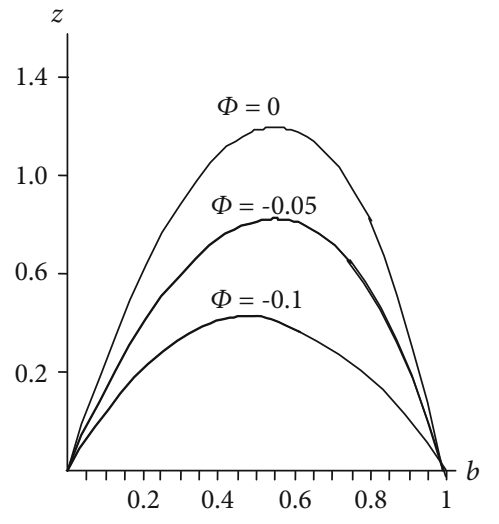

Figure 10: Velocity of $Z$ when $N=0.5, R=2.5$.

TABLE 1: Numerical values of $\psi$ and $R_{z}$ for various values of $A, N, q$, $R$, and $j i^{\prime}$ with $j=1.0, y=1.0, L=0.7, \mu=1.0, f_{g}=1.0$, and $\varepsilon=0.1$.

\begin{tabular}{ccccccc}
\hline$A$ & $N$ & $q$ & $R$ & $j i^{\prime}$ & $\psi$ & $-R_{z}$ \\
\hline 1.0 & 1.0 & 1.0 & 1.0 & $\pi / 4$ & 0.17735 & 0.70064 \\
2.0 & & & & & 0.12671 & 0.70064 \\
3.0 & & & & & 0.09856 & 0.70064 \\
& 1.0 & 1.0 & 1.0 & $\pi / 4$ & 0.17735 & 0.70064 \\
1.0 & 2.0 & & & & 0.15448 & 0.70064 \\
& 3.0 & & & & 0.12680 & 0.70064 \\
& 2.0 & 1.0 & 1.0 & $\pi / 4$ & 0.15448 & 0.70064 \\
1.0 & & 2.0 & & & 0.13666 & 0.70064 \\
& & 3.0 & & & 0.11431 & 0.70064 \\
& 2.0 & 1.0 & 0.5 & $\pi / 4$ & 0.17292 & 0.61824 \\
1.0 & & & 1.0 & & 0.17735 & 0.70064 \\
& & & 1.5 & & 0.18660 & 0.87674 \\
& 1.0 & 1.0 & 1.0 & $\pi / 4$ & 0.17735 & 0.70064 \\
& & & & $\pi / 2$ & 0.44549 & 1.06665 \\
1.0 & & & & $3 \pi / 4$ & 0.55036 & 1.1620 \\
& & & & $\pi$ & 0.57605 & 1.18286 \\
\hline
\end{tabular}

reduces the velocity profile and the fluid velocity is parabolic within maximum value along the centerline of the channel and minimum at the walls. Nevertheless, we observed that the fluid velocity profile increases when there is an increase in the frequency parameter, and the increase of nonNewtonian fluid parameter reduces the skin friction coefficient, while the frequency parameter increases the wall heat transfer rate. Also, it was interesting to compare the results with the corresponding flow problems for a Newtonian fluid.

\section{Abbreviations}

$z: \quad$ The axial velocity

$i$ : $\quad$ The time

$\lambda: \quad$ The fluid density

$u$ : $\quad$ The pressure

७: $\quad$ Kinematic viscosity coefficient

$v$ : Coefficient of viscoelasticity
$P: \quad$ The porous medium permeability

$\rho: \quad$ Conductivity of the fluid

$E_{0}=\gamma I$ : The electromagnetic induction

$\gamma: \quad$ The magnetic permeability

I: $\quad$ The intensity of the magnetic field

$h$ : The gravitational force

$r: \quad$ The fluid temperature

$r_{0}: \quad$ Temperature at $b=0$

$r_{j}: \quad$ Temperature at $b=m$

$\alpha$ : $\quad$ The coefficient of volume expansion due to temperature

S: $\quad$ Specific heat at constant pressure

$v: \quad$ The radiative heat flux

$t$ : Thermal conductivity.

\section{Data Availability}

No data were used to support this study.

\section{Consent}

There was no objection after informing me to agree to its publication.

\section{Conflicts of Interest}

The author declares that she has no competing interests.

\section{Acknowledgments}

This research was funded by the Deanship of Scientific Research at Princess Nourah Bint Abdulrahman University through the Fast-track Research Funding Program.

\section{References}

[1] N. A. Alessa, "Lie group analysis for heat generation and radiation effects on porous nanofluid flow," Journal of Computational and Theoretical Nanoscience, vol. 14, no. 11, pp. 52925297, 2017.

[2] J. Olver, Application of Lie Groups to Differential Equations, Springer, New York, NY, USA, 1989.

[3] R. Moreau, Magnetohydrodynamics, Kluwer Academic Publishers, Dordrecht, 1990.

[4] Y. J. Kim, "Unsteady MHD convective heat transfer past a semi-infinite vertical porous moving plate with variable suction," International Journal of Engineering Science, vol. 38, no. 8, pp. 833-845, 2000.

[5] A. R. Rao and K. S. Deshikachar, "MHD oscillatory flow of blood through channels of variable cross section," International Journal of Engineering Science, vol. 24, no. 10, pp. 1615-1628, 1986.

[6] O. D. Makinde, "Radiation effects on free convection flow in a channel of slowly varying width," AMSE Modelling, Measurement and Control B, vol. 70, no. 2, pp. 9-23, 2001.

[7] O. D. Makinde, "Magneto-hydrodynamic stability of planePoiseuille flow using multideck asymptotic technique," Mathematical and Computer Modelling, vol. 37, no. 3-4, pp. 251259, 2003. 
[8] R. P. Agarwal, S. Gala, and M. A. Ragusa, "A regularity criterion in weak spaces to Boussinesq equations," Mathematics, vol. 8, no. 6, p. 920, 2020.

[9] S. Benbernou, S. Gala, and M. A. Ragusa, "On the regularity criteria for the 3D magnetohydrodynamic equations via two components in terms of BMO space," Mathematical Methods in the Applied Sciences, vol. 37, no. 15, pp. 2320-2325, 2014.

[10] O. D. Makinde, "MHD steady flow and heat transfer on the sliding plate," AMSE-Modelling, Simulation \& Control, vol. 70, no. 1, pp. 61-70, 2001.

[11] T. Hayat and Y. Wang, "Magnetohydrodynamic flow due to noncoaxial rotations of a porous disk and a fourth-grade fluid at infinity," Mathematical Problems in Engineering, vol. 2003, Article ID 674518, 18 pages, 2003.

[12] O. D. Makinde and P. Sibanda, "On suction driven flow and heat transfer in a pipe filled with porous media," Computer Assisted Methods in Engineering and Science, vol. 5, pp. 389398, 1998.

[13] A. J. Chamkha, "The Stokes problem for a dusty fluid in the presence of magnetic field, heat generation and wall suction effects," International Journal of Numerical Methods for Heat \& Fluid Flow, vol. 10, no. 1, pp. 116-133, 2000.

[14] A. Raptis, C. Massalas, and G. Tzivanidis, "Hydromagnetic free convection flow through a porous medium between two parallel plates," Physics Letters A, vol. 90, no. 6, pp. 288-289, 1982.

[15] T. K. Aldoss, M. A. Al-Nimr, M. A. Jarrah, and B. Al-Shaer, "Mixed convection flow from a vertical plate embedded in porous medium in the presence of magnetic field," Heat Transfer, vol. 28A, pp. 635-645, 1995.

[16] O. D. Makinde and P. Y. Mhone, "Heat transfer to MHD oscillatory flow in a channel filled with porous medium," Romanian Journal of Physics, vol. 50, no. 9-10, pp. 931-938, 2005.

[17] A. C. L. Cogley, W. G. Vincent, and E. S. Giles, "Differential approximation for radiative transfer in a nongrey gas near equilibrium," American Institute of Aeronautics and Astronautics, vol. 6, pp. 551-553, 1968.

[18] D. P. Mason, "Group invariant solution and conservation law for a free laminar two-dimensional jet," Journal of Nonlinear Mathematical Physics, vol. 9, Supplement 2, pp. 92-101, 2013. 